\title{
Ap stars as progenitors of magnetic white dwarfs
}

\author{
A. Kawka ${ }^{1}$ and S. Vennes ${ }^{2}$ \\ ${ }^{1}$ Astronomický ústav AV ČR, Fričova 298, 25165 Ondřejov, Czech Republic \\ ${ }^{2}$ Department of Physics and Astronomy, 3400 North Charles Street, Johns Hopkins University, \\ Baltimore, MD 21218, USA
}

\begin{abstract}
The progenitors of magnetic white dwarfs are believed to be magnetic Ap and Bp stars because the fields in these stars are structured and are present in the stellar core. As in Ap/Bp stars the magnetic fields in white dwarfs are, in most cases, dipolar or quadrupolar with various offsets. Although the present space density of Ap/Bp progenitors would be sufficient to account for the density of magnetic white dwarfs in young populations such as found in the Palomar-Green survey, we show that it would be insufficient to generate the density of known magnetic white dwarfs in the older solar neighborhood. Assuming magnetic flux conservation during the final stages of evolution, we find that $\mathrm{Ap} / \mathrm{Bp}$ stars would evolve into white dwarfs with magnetic fields exceeding $10^{7} \mathrm{G}$, assuming a minimum polar field of $200 \mathrm{G}$ in Ap/Bp stars, thereby leaving many magnetic white dwarfs with lower fields without likely progenitors.
\end{abstract}

Keywords. Stars: magnetic fields, white dwarfs, evolution

\section{Introduction}

The progenitors of magnetic white dwarfs are assumed to be Ap/Bp stars (referred to as Ap stars from hereon). Indeed, the magnetic fields of white dwarfs are structured like those of Ap stars. Magnetic fields in white dwarfs are usually centered or offset dipoles or quadrupoles, with a few of them having irregular features such as a magnetic spot.

Angel et al. (1981) were the first to compare the space density of magnetic white dwarfs to the predicted space density of magnetic core remnants of Ap stars, i.e., the white dwarf descendants of Ap stars. They have taken the space density of the magnetic white dwarfs to be the density of known magnetic white dwarfs within $15 \mathrm{pc}$ of the Sun $\left(5.3 \pm 2.3 \times 10^{-4} \mathrm{pc}^{-3}\right)$. They assumed a density of $\sim 1-2 \times 10^{-5} \mathrm{pc}^{-3}$ for Ap stars, where they assumed that all Ap stars have magnetic fields larger than $100 \mathrm{G}$. To calculate the predicted density of magnetic core remnants, they used the Main-Sequence lifetime of a $3.6 M_{\odot}$ star $\left(\sim 1.2 \times 10^{8} \mathrm{yr}\right)$ and assumed that the production rate of these stars has been constant during the past $5 \times 10^{9} \mathrm{yr}$. Therefore, they predicted the density of magnetic core remnants to be in the range of $4-8 \times 10^{-4} \mathrm{pc}^{-3}$ close to the observed number. They suggest that this result could vary, since the formation rate of stars may have been higher in the past.

Given that the origin and the properties of magnetic Ap stars still remain uncertain, the assumptions made by Angel et al. (1981) should be revisited using more recent results. In particular, the details of the predicted and observed field distributions should be reconsidered. Therefore, we will examine whether the assumption that Ap stars are the sole progenitors of the observed population of magnetic white dwarfs is valid. We will first review the properties of magnetic white dwarfs, in particular their space density and magnetic field distribution. Next, we will review the properties of Ap stars, and follow their evolution into white dwarf stars. We will conclude with a comparison of the observed and predicted space density and field distribution of magnetic white dwarf stars. 


\section{Magnetic white dwarfs}

Magnetic fields in white dwarfs range from a kilogauss or less to close to a billion gauss, as evidenced in recent Sloan Digitized Sky Survey discoveries (Schmidt et al. 2003). Figure 1 shows the spectra of five of the Sloan magnetic white dwarfs with increasing field strengths. Schmidt \& Smith (1995) used their survey criteria to find that the fraction of magnetic white dwarfs is $4 \pm 1.5 \%$ which, scaled to the local white dwarf space density, i.e., $5.0 \pm 0.7 \times 10^{-3} \mathrm{pc}^{-3}$ (Holberg et al. 2002), corresponds to a space density of $\sim 2 \times 10^{-4} \mathrm{pc}^{-3}$. However, the fraction of magnetic white dwarfs in the Solar Neighborhood is now estimated at $17 \pm 6 \%$ (Kawka et al. 2003), corresponding to a space density of $8.5 \times 10^{-4} \mathrm{pc}^{-3}$.

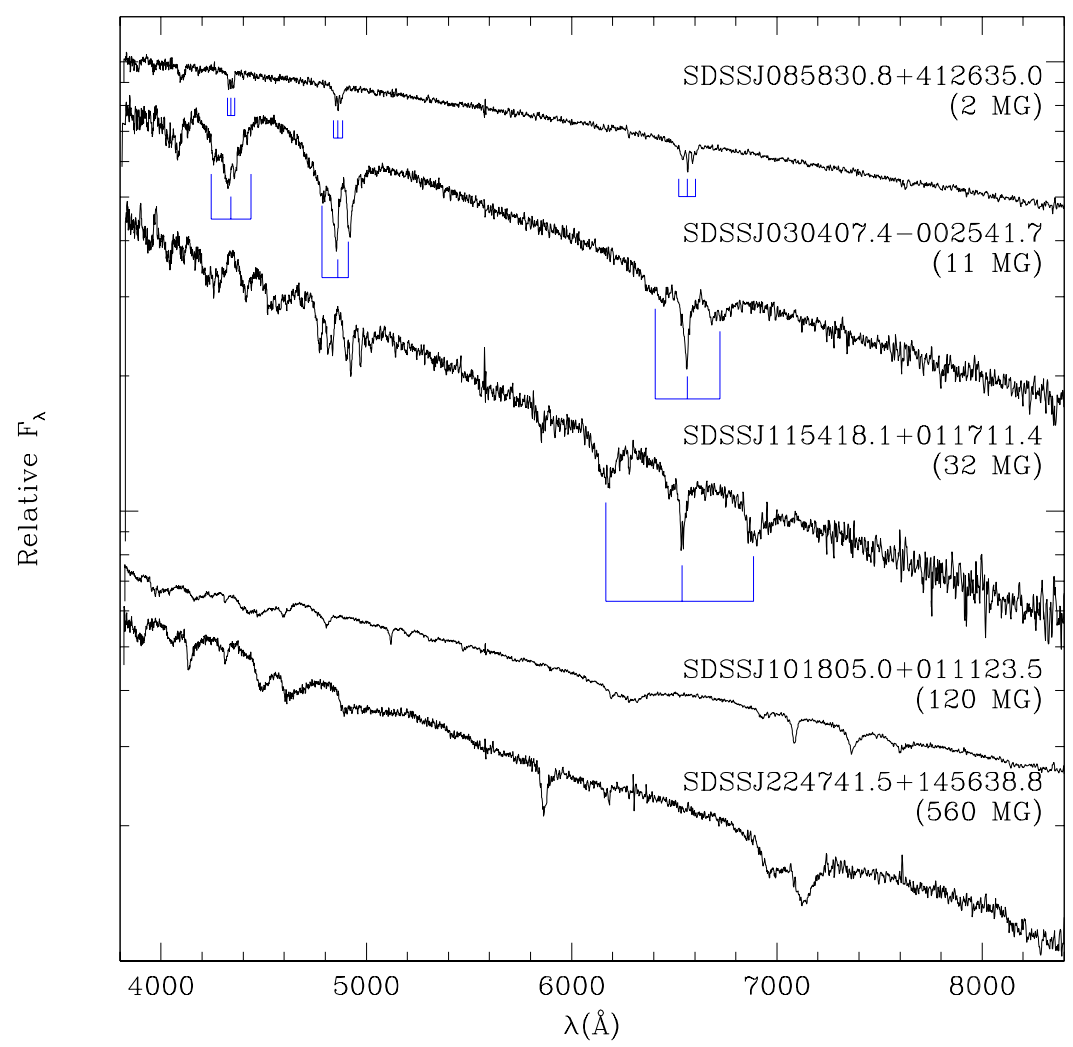

Figure 1. Examples of five magnetic white dwarfs that were observed as part of the Sloan Digital Sky Survey (Schmidt et al. 2003). The Zeeman splitting is observed in the weaker field stars and the highly shifted Zeeman components in the strongly magnetic white dwarfs. The lower-field spectra are compared to the predicted Zeeman components (Kemic 1974).

The local population of white dwarfs is considered to be old, compared to other samples of white dwarfs for which the incidence of magnetism has been determined, and the incidence of magnetism in white dwarfs appears higher among older white dwarfs (Liebert \& Sion 1979; Valyavin \& Fabrika 1999) indicating a larger fraction of magnetic stars in the parent population of old white dwarfs. Liebert et al. (2003) have used the list of local white dwarfs from Holberg et al. (2002) and found that $16 \%$ of stars below $8000 \mathrm{~K}$ are magnetic compared to only $3 \%$ of stars hotter than this temperature. For instance, Liebert et al. (2003) found that $2 \pm 0.8 \%$ of the white dwarfs in the Palomar-Green $(\mathrm{PG})$ survey are magnetic. However, given that the survey is magnitude limited, they 
suggests that a radius bias against the detection of magnetic white dwarfs may exist, if one assumes that the mean mass of magnetic white dwarfs is higher than for nonmagnetic white dwarfs. Correcting for this radius bias, they suggest that the incidence of magnetism is more like $8 \pm 3 \%$, still only half the density of the cooler sample.

The field distribution of all known magnetic white dwarfs peaks near $10^{7} \mathrm{G}$ is probably a result of a spectroscopic selection of magnetic white dwarfs with obvious Zeeman splitting (see Fig. 1). Low-field and high-field white dwarfs are less conspicuous. Kawka et al. (2003) estimate that the field distribution in the local population of white dwarfs is flat, with the fraction of magnetic white dwarfs roughly a constant irrespective of field strength, in agreement with the analysis of Schmidt \& Smith (1995).

\section{From magnetic Ap stars to white dwarfs}

The field distribution in the Ap stars is not well established. In separate efforts aimed at opposite ends of the field distribution, Mathys et al. (1997) and Hubrig et al. (2000) surveyed the properties of Ap stars with obvious or suspected Zeeman line splitting while Aurière \& Wade (2005) obtained polarimetric spectra of a sample of Ap stars with small or undetected fields. Combining both studies indicates that polar fields $B_{\mathrm{p}}$ in Ap stars may range from $200 \mathrm{G}$ to over $20000 \mathrm{G}$. Overall, Bychkov et al. (2003) estimates that the longitudinal field distribution declines exponentially with field strength following a relation of the form $N \propto \mathrm{e}^{-B_{\mathrm{e}} / 789.2 \mathrm{G}}$.
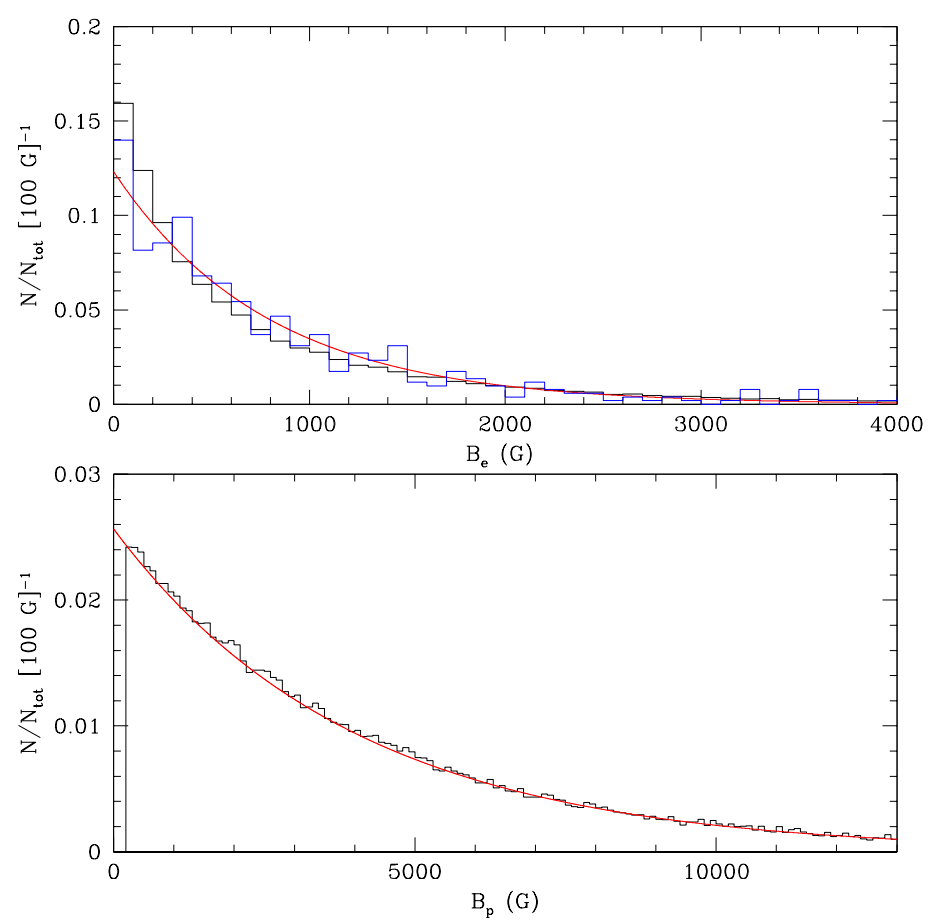

Figure 2. (Bottom) Randomly generated $B_{\mathrm{p}}$ field distribution following exponential deviates (Press et al. 1992) compared to an exponential function $N \propto \mathrm{e}^{-B_{\mathrm{p}} / 4000 \mathrm{G}}($ red). (Top) Simulated $B_{e}$ field distribution (black) using the randomly generated $B_{\mathrm{p}}$ field distribution converted into longitudinal fields using randomly generated angles, compared to an exponential function $N \propto \mathrm{e}^{-B_{\mathrm{e}} / 789.2 \mathrm{G}}(r e d)$ and the observed distribution (blue). 
Following Monte Carlo simulation techniques the magnetic field distribution of Ap stars was calculated using exponential deviates (Press et al. 1992):

$$
B_{\mathrm{p}}=-4000 \log n
$$

where $n$ is a randomly generated number between 0 and 1 (exclusive of endpoints). The resulting distribution assembles 100000 runs and has the shape of an exponential function. The polar fields are then converted into longitudinal fields using $B_{\mathrm{e}}=0.4 B_{\mathrm{p}} \cos i$, where $i$ is randomly generated between 0 and $90^{\circ}$ weighted by $\sin i$. Figure 2 shows that a distribution of polar fields which follows a relation of the form $N \propto \mathrm{e}^{-B_{\mathrm{p}} / 4000 \mathrm{G}}$, where $B_{\mathrm{p}}>200 \mathrm{G}$, may be converted into a similar relation which closely follows the prescription of Bychkov et al. (2003).

For each of the $100000 B_{\mathrm{p}}$ runs, we randomly generated a mass using Gaussian deviates (Press et al. 1992). Therefore, we neglected a possible field/spectral type correlation. The Gaussian parameters, $\left(M, \sigma_{M}\right)=(2.5,0.4)$, were determined empirically from the mass distribution for the population of Ap stars built from the measured $B-V$ distribution (Johnson 2004; Renson et al. 1991) and the $B-V$ versus mass and radius tabulations of Gray (1992). The resulting mass distribution encompasses the sample of Hubrig et al. (2000) which does not differ significantly from the mean. Then, we allow evolution to take place. The Ap masses were converted into remnant core masses using initial-to-final mass relations with solar metallicity from Dominguez et al. (1999), although these initial-tofinal mass relations may not be applicable to magnetic stars. The radii of the remnant cores were calculated using the white dwarf mass-radius relations of Wood (1995). Since the average temperature of magnetic white dwarfs is about $15000 \mathrm{~K}$ and the majority of them having a hydrogen-rich atmosphere, the models at $T_{\text {eff }}=15000 \mathrm{~K}$ with a thick hydrogen atmosphere were used. Next, the predicted magnetic field of the remnant core was determined using the calculated radii, the estimated polar field strength of the Ap star, and the assumption that magnetic flux is conserved $\left(B R^{2}=\right.$ constant). Finally, a magnetic field distribution of the remnant cores was built by assembling together the predicted fields.

At this stage only the relative magnetic field distribution of Ap remnant cores has been determined. The distribution requires normalization to the predicted space density. The expected number of magnetic white dwarfs can be calculated using the luminosity function for Main-Sequence stars.

Given that Ap stars have spectral types ranging from F0 to B2 (Wade 2001), their absolute magnitudes range from 2.5 to -2.5 (see Houk et al. 1997 and (Murray et al. 1997). The luminosity function suggests a current F0-B2 space density of $2.9 \times 10^{-4}$ $\mathrm{pc}^{-3}$ in the Solar Neighborhood (Murray et al. 1997). About $10 \%$ of these objects are magnetic Ap stars (Moss 2001) (i.e., all Ap stars are magnetic), hence the space density of magnetic Ap stars is $\approx 3 \times 10^{-5} \mathrm{pc}^{-3}$.

Next, a production rate of Ap remnant cores can be obtained by dividing the MainSequence luminosity function of Murray et al. 1997 by the Main-Sequence lifetimes of Dominguez et al. (1999) for each luminosity bin. The lifetime of a star on the MainSequence is the central H-burning lifetime. As a simple test, we integrated over all absolute magnitudes to obtain the number of white dwarfs formed in the past $7 \times 10^{9}$ years. Assuming a constant Main-Sequence luminosity function, we found an estimated space density of white dwarfs to be $5 \times 10^{-3} \mathrm{pc}^{-3}$, in agreement with current estimates. Integrating over absolute magnitudes from 2.5 to -2.5 and accounting for $10 \%$ incidence of magnetism in this magnitude range, we estimate that $4.3 \%$ of white dwarfs are formed from magnetic Ap stars. Angel et al. (1981) obtained a much larger percentage of predicted magnetic white dwarfs because of a shorter H-burning time-scale of $1.2 \times 10^{8}$ 
years and larger average mass of $3.6 M_{\odot}$ adopted for Ap stars. In our calculations, the average mass of Ap stars is $\approx 2.5 M_{\odot}$ which corresponds to a time-scale of $\approx 5 \times 10^{8}$ years, resulting in a much lower production rate. The production rate can be increased by $30 \%$ using the Main-Sequence lifetimes of Dominguez et al. (1999) for low-metallicity stars $\left(Z=6 \times 10^{-3}\right)$, more typical of earlier generations of stars, instead of the solar metallicity models.

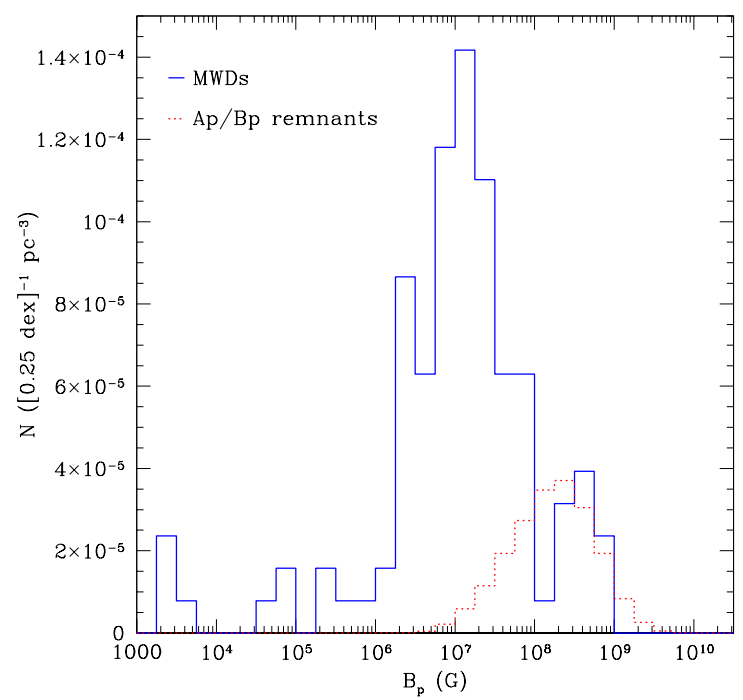

Figure 3. The measured magnetic white dwarf incidence (full line) compared to the predicted magnetic incidence of Ap remnants (dotted line).

Figure 3 shows the observed field distribution normalized to the observed space density of magnetic white dwarfs (assuming that $17 \%$ of white dwarfs are magnetic) compared to the predicted field distribution of magnetic Ap remnants normalized to the predicted space density of magnetic Ap remnants $\left(2 \times 10^{-4} \mathrm{pc}^{-3}\right)$. Note that the observed field distribution in the sample of all known magnetic white dwarfs peaks at $\approx 3 \times 10^{7} \mathrm{G}$, unlike the field distribution in the local sample which appears flat (Kawka et al. 2003). However, the recent discovery of three magnetic white dwarfs with weak fields $(<10 \mathrm{kG})$ by Aznar Cuadrado et al. (2004) may imply that a substantial fraction of white dwarfs may have a weak magnetic field. Aznar Cuadrado et al. (2004) suggest that this could be as high as 25\%. High signal-to-noise studies, such as that of Aznar Cuadrado et al. (2004) are required to determine the weak-field distribution of magnetic white dwarfs. The known sample is possibly biased toward mid-strength fields which are more readily identified in low-resolution spectroscopy.

\section{Summary and discussion}

We have calculated the magnetic field distribution of the remnant Ap stellar cores and compared this distribution to the present field distribution of magnetic white dwarfs. The two distributions were normalized to the predicted space density of Ap remnants, and to the observed space density of local magnetic white dwarfs, respectively. We found that Ap stars do not generate a sufficient number of magnetic white dwarfs and that Ap stars only generate white dwarfs with magnetic fields exceeding $10^{7}$ G. Therefore, 
additional progenitors are required for low-field white dwarfs. These progenitors could be Main-Sequence stars with low-fields $(\leqslant \sim 200 \mathrm{G})$ that have eluded detection.

There are a number of unresolved issues in performing such calculations. One is that the average mass of magnetic white dwarfs appears higher than predicted by theory, $\sim 0.9 M_{\odot}$ versus $\sim 0.6 M_{\odot}$. The average mass of the remnant cores of Ap stars in our calculations is $\sim 0.6 M_{\odot}$, close to the observed mass of normal nonmagnetic white dwarfs. Note that A and B stars do not normally produce white dwarfs with masses $\sim 0.9 M_{\odot}$ when standard initial-to-final mass relations are used. One suggestion for creating more massive remnant cores is that magnetic fields may inhibit mass loss during the final stages of evolution. The magnetic white dwarf, EG 61, in the Praesepe cluster is an example for which this scenario was suggested. The mass of EG 61 is $0.9 M_{\odot}$, and is believed to have evolved from a Main-Sequence star of $2.2 M_{\odot}$ (Kanaan et al. 1999). Using initial-to-final mass relations, the expected mass of a $2.2 M_{\odot}$ star would be about $0.5-0.6 M_{\odot}$.

Magnetic flux may not be conserved in the final stages of evolution after all, and some magnetic flux may be lost along with the stellar envelope. Therefore, lower fields could be created in the remnant cores. However, the predicted spatial density of remnant Ap stellar cores would remain too low, particularly in the local white dwarf population. A higher formation rate of Ap stars in the younger Galaxy would allow for a larger incidence of cool, hence old magnetic white dwarfs. Drastically different scenarios for the higher incidence of cooler magnetic white dwarfs were proposed by Valyavin \& Fabrika (1999). They suggest that white dwarfs may experience an increase in electric conductivity with age, allowing magnetic fields to develop, or alternatively that magnetic fields may diffuse from the inner regions outward, after field lines were buried by the collapse of the core during the red giant phase. The magnetic field would diffuse outward with time.

The above scenarios require that magnetic fields in Ap stars be present in the core and survive, in whole or in part, through the final stages of evolution. The two leading proposals for the origin of magnetic fields in Ap stars are the dynamo and fossil field theories. Both models experience difficulties in explaining the properties of magnetic fields in Ap stars (see Moss (2001) for a review), and also present different outcomes for white dwarf magnetic fields. In the core-dynamo theory, the observed fields are those in the convective and rotating cores that have slowly diffused toward the surface. The main problem with this theory is that, contrary to observations, all A and B stars should display surface magnetic fields, and not just Ap stars. The theory may lead to predict an excessive number of magnetic white dwarfs! On the contrary, the leading problem with the the fossil field theory, is that the magnetic flux may not survive pre-Main Sequence evolution. However, assuming fields do survive, then the theory may offer a natural explanation for the weakness or lack of magnetic fields in most white dwarfs. In this theory, the magnetic field is not generated in the core, therefore, much of the magnetic flux in the envelope is lost during the mass-loss phase, leaving only a fraction of the original Ap star flux behind. Much about these questions is still debated.

We thank D. Moss and G. Wade for their comments and suggestions.

\section{References}

Angel, J.R.P., Borra, E.F., \& Landstreet, J.D. 1981, ApJS, 45, 457

Aurière, M., et al. 2005, These Proceedings, EP12

Aznar Cuadrado, R., Jordan, S., Napiwotzki, R., Schmid, H.M., Solanki, S.K. \& Mathys, G. 2004, A\&A, 423, 1081

Bychkov, V.D., Bychkova, L.V., \& Madej, J. 2003, A\&A, 407, 631

Dominguez, I., Chieffi, A., Limongi, M. \& Straniero, O. 1999, ApJ, 524, 226 
Gray, D.F. 1992, The observation and analysis of stellar atmospheres, Cambridge University Press

Holberg, J.B., Oswalt, T.D. \& Sion, E.M. 2002, ApJ, 571, 512

Houk, N., Swift, C.M., Murray, C.A., Penston, M.J. \& Binney, J.J. 1997, ESA SP-402: Hipparcos - Venice '97, 279

Hubrig, S., North, P. \& Mathys, G. 2000, ApJ, 539, 352

Johnson, N. 2004, M.Sc. Dissertation, Royal Military College of Canada

Kanaan, A., Claver, C.F., \& Liebert, J. 1999, in ASP Conf. Ser. 169: 11th European Workshop on White Dwarfs, eds. Solheim, J.E. \& Meistas, E.G., 221

Kawka, A., Vennes, S., Wickramasinghe, D.T., Schmidt, G.D. \& Koch, R. 2003, in White Dwarfs, NATO Science Series II, Vol. 105, eds. de Martino, D., Silvotti, R., Solheim, J.-E., Kalytis, R., 179

Kemic, S.B. 1974, JILA REP. No. 113

Liebert, J., Bergeron, P. \& Holberg, J.B. 2003, AJ, 125, 348

Liebert, J. \& Sion, E.M. 1979, textitAstrophys. Letters, 20, 53

Mathys, G., Hubrig, S., Landstreet, J.D., Lanz, T. \& Manfroid, J. 1997, A\&AS, 123, 353

Moss, D. 2001, ASP Conf. Ser. 248: Magnetic Fields Across the Hertzsprung-Russell Diagram, eds. Mathys, G.A., Solanki, S.K. \& Wickramasinghe, D.T., 305

Murray, C.A., Penston, M.J., Binney, J.J. \& Houk, N. 1997, ESA SP-402: Hipparcos - Venice ' 97,485

Press, W. H., Teukolsky, S. A., Vetterling, W. T., \& Flannery, B. P. 1992, Cambridge University Press

Renson, P., Gerbaldi M., \& Catalano, F.A. 1991, A\&AS, 89, 429

Schmidt, G.D., et al. 2003, ApJ, 595, 1101

Schmidt, G.D. \& Smith, P.S. 1995, ApJ, 448, 305

Valyavin, G. \& Fabrika, S. 1999, in ASP Conf. Ser. 169: 11th European Workshop on White Dwarfs, eds. Solheim, J.E. \& Meistas, E.G., 206

Wade, G.A. 2001, ASP Conf. Ser. 248: Magnetic Fields Across the Hertzsprung-Russell Diagram, eds. Mathys, G.A., Solanki, S.K. \& Wickramasinghe, D.T., 403

Wood, M.A. 1995, in White Dwarfs (New York: Springer), eds. Koester, D. \& Werner, K., 41 